Article

\title{
Social Exclusion of Australian Childless Women in Their Reproductive Years
}

\author{
Beth Turnbull *, Melissa L. Graham and Ann R. Taket \\ Centre for Health through Action on Social Exclusion (CHASE), School of Health and Social Development, \\ Deakin University, Burwood, VIC 3125, Australia; E-Mails: b.turnbull@deakin.edu.au (B.T.), \\ melissa.graham@deakin.edu.au (M.L.G.), ann.taket@deakin.edu.au (A.R.T.) \\ * Corresponding author
}

Submitted: 27 October 2015 | Accepted: 16 January 2016 | Published: 29 February 2016

\begin{abstract}
Research suggests Australian childless women are at risk of pronatalism-driven social exclusion. This exploratory, mixed methods, cross-sectional study described and explored the social exclusion of Australian childless women aged 25 to 44 years, and asked: what are the nature and extent of social exclusion of childless women; and do the nature and extent of exclusion vary for different types of childless women? A total of 776 childless female Australian residents aged 25 to 44 years completed a self-administered questionnaire. Quantitative data were collected on childlessness types, indicators of exclusion and perceived stigmatisation and exclusion due to being childless. Data were analysed using descriptive statistics, One Way ANOVAs and Kruskal Wallis Analysis of Ranks. Qualitative data on childless women's experiences were inductively thematically analysed. Findings suggest societal-level pronatalism drives exclusion of Australian childless women. While exclusion occurs in all domains of life, childless women experience more exclusion, and perceive more exclusion due to being childless, in the social and civic domains than the service and economic domains. Circumstantially and involuntarily childless women, followed by voluntarily childless women, perceive more exclusion due to being childless than undecided and future childed women. Experiences are influenced by the nature of women's 'deviance' from pronatalism.
\end{abstract}

\section{Keywords}

childlessness; demography; fertility; pronatalism; social exclusion; stereotypes; stigma

Issue

This article is part of a regular issue of Social Inclusion, edited by Professor Ulf R. Hedetoft (University of Copenhagen, Denmark).

(C) 2016 by the authors; licensee Cogitatio (Lisbon, Portugal). This article is licensed under a Creative Commons Attribution 4.0 International License (CC BY).

\section{Introduction}

Increasing numbers of Australian women aged between 25 and 44 years have no biological children, rising from 33 per cent in 2006 to 35.5 per cent in 2011 (Australian Bureau of Statistics [ABS], 2007, 2012a). Research suggests pronatalism in Australian society places childless women at risk of social exclusion (see for example Graham \& Rich, 2012a; Sawer, 2013).

Social exclusion is a multidimensional process driven by unequal power relationships interacting between the societal, community, relationship and individual levels, and manifesting in a continuum of inclusion and exclusion characterised by constraints upon the extent and quality of resources and opportunities for participation in the social, civic, service and economic domains of life (Levitas et al., 2007; Popay et al., 2008). Research suggests societal-level pronatalism drives stigmatisation and exclusion of childless women. Pronatalism constructs women as mothers, manifesting at a societal level in policies, discourses and ideologies designed to promote fertility by representing mother- 
hood as a moral, patriotic and economic duty, and at community, relationship and individual levels in cultures, beliefs, attitudes and behaviours that assume motherhood is natural, innate and inevitable (Gillespie, 2000; Graham \& Rich, 2012a; Veevers, 1979). By constructing motherhood as central to being a woman, pronatalism perpetuates patriarchal power by disempowering women as resource-less mothers, and marginalising 'deviant' childless women (Gillespie, 2000; Park, 2002).

Political and media analyses have revealed hegemonic pronatalist discourses in Australian society constructing childed women as idealised 'insiders' and childless women as stigmatised 'outsiders' (Graham \& Rich, 2012a, 2012b; Sawer, 2013). Canadian and United States studies found most adults believed motherhood was innate and natural (Miall, 1994), and more adults had negative or neutral than positive attitudes towards being childless (Koropeckyj-Cox \& Pendell, 2007). Research from Australia, the United Kingdom and United States found women experienced being childless as discrediting, and believed others perceived childless women as unnatural, deficient, unfulfilled and incomplete (Letherby, 1999; Rich, Taket, Graham, \& Shelley, 2011; Sternke \& Abrahamson, 2015). Some involuntarily childless women internalised pronatalism, perceiving themselves as failures and incomplete (Bell, 2013; Sternke \& Abrahamson, 2015). There is, however, limited research investigating the exclusion of childless women in their reproductive years from the social domain, which encompasses social networks, support and interaction, and social and leisure participation (see for example, Albertini \& Mencarini, 2014; Bell, 2013; Debest \& Mazuy, 2014; Doyle, Pooley, \& Breen, 2013; McNamee \& James, 2012; Sternke \& Abrahamson, 2015), the service domain, which incorporates availability, accessibility, affordability, appropriateness and adequacy of services (see for example Baker, 2003; Hammarberg, Astbury, \& Baker, 2001; Mollen, 2006; Onat \& Beji, 2012; Parry, 2004) and the economic domain, including participation in employment, the nature and quality of working lives, and material and financial resources (see for example Doyle et al., 2013; Fieder, Huber, \& Bookstein, 2011; Huber, Bookstein, \& Fieder, 2010; Malik \& Coulson, 2013; Rich et al., 2011). There is no existing research in the civic domain, which includes participation in community and political activities, groups and organisations.

While the extant evidence reveals the complexity and diversity of childless women's experiences, it emerges from disparate studies investigating distinct domains of life and undifferentiated or particular types of childless women. There is no Australian research investigating social exclusion of different types of childless women in their reproductive years from multiple domains of life. This exploratory study aimed to describe and explore the social exclusion of Australian childless women aged 25 to 44 years, and asked: (1) what are the nature and extent of social exclusion of childless women; and (2) do the nature and extent of social exclusion vary for different types of childless women?

While acknowledging the negative connotations associated with terms used to describe women with no children, 'childless' and 'childed' are used to describe women without and with children respectively in this paper.

\section{Methods}

The study was conducted in Australia during 2014. Ethics approval was obtained from Deakin University's Human Ethics Advisory Group (HEAG-H 175_2013). The cross-sectional, mixed-methods study employed a fully mixed concurrent equal status design (Leech \& Onwuegbuzie, 2009).

\subsection{Sampling}

The target population was childless female Australian residents aged 25 to 44 years who were not pregnant and identified as never having assumed the role or identity of a biological or social mother, for example, of adopted, step or fostered-children. The age range encompassed Australian women's mean age of first birth of 28 years, peak reproductive age of 30 to 34 years, and usual completion of fertility between 40 and 44 years (ABS, 2008, 2010a; Australian Institute of Health and Welfare, 2010).

The formula for a binomial distributions k-sample for the chi-square test (Brown et al., 2010) yielded a minimum sample size of 427 , including 171 voluntarily childless women, who freely chose not to have children (Veevers, 1979), 64 involuntarily childless women, who wished to have biological children but were unable to achieve a viable pregnancy (Daniluk, 2001), 64 circumstantially childless women, who were unable to have children due to other circumstances such as having no partner, partner infertility, or health issues other than those preventing a viable pregnancy (Cannold, 2005), 64 undecided women, who were unsure about whether to have children, and 64 future childed women, who intended to have biological or social children in future.

In the absence of a sampling frame, participants were recruited by promoting the study through 38 women's health services, 13 blogs and Facebook pages, professional networks and snowball sampling. Of the 1,411 respondents, 631 women who did not click the 'submit' button at the end of the questionnaire were excluded pursuant to the ethics approval as deemed withdrawals; and four whose dates of birth fell outside the target age range were excluded. The total sample size was $\mathbf{7 7 6}$ women, a completion rate of fifty-five per cent. 


\subsection{Data Collection and Measures}

A self-administered online questionnaire collected quantitative data on socio-demographic variables, types of childlessness and social exclusion. Indicators of exclusion were measured using the social interaction subscale of the Duke Social Support Index (George, Blazer, Hughes, \& Fowler, 1989) and the MOS social support index (Chronbach's $\alpha=0.97$; one-year stability $\alpha=0.78$ ) (Sherbourne \& Stewart, 1991) in the social domain, participation in community groups, activities and events (ABS, 2010b) in the civic domain, degree of problems accessing and using services (Dermott et al., 2012) in the service domain, and employment status (Melbourne Institute of Applied Economic and Social Research, 2012) and personal income (ABS, 2011) in the economic domain. Data were also collected on stigma consciousness (Chronbach's $\alpha=0.72-0.74$ ) (Pinel, 1999) and degree of perceived exclusion from domains of life due to being childless. Open-ended questions employing the critical incident technique, which seeks to obtain detailed accounts of participants' experiences of past incidents (Butterfield, Borgen, Amundson, \& Maglio, 2005), gathered qualitative data on each domain (see Appendix).

\subsection{Data Analysis}

Aggregate scores were computed for social interaction, social networks, social support, community participation, problems accessing and using services and stigma consciousness (Hughes, Blazer, \& Hybels, 1990; Sherbourne \& Stewart, 1991). Employment status was computed from items on paid work status, underemployment and unemployment. Personal income ranges were recoded into income levels (ABS, 2012c). Fivepoint scales measuring degree of perceived exclusion due to being childless were collapsed into binary ordinal variables consisting of no perceived exclusion or at least a slight degree of perceived exclusion.

Descriptive statistics examined socio-demographic characteristics, indicators of exclusion and stigmatisation and exclusion due to being childless. One Way ANOVAs and Scheffe's post-hoc tests analysed differences between types of childless women's stigma consciousness. Kruskal Wallis $\mathrm{H}$ and Dunn's post-hoc tests analysed differences in social interaction, social support, community participation, problems accessing or using services, employment status, personal income and perceived exclusion due to being childless.

Qualitative data were inductively thematically analysed through data immersion by reading and rereading all qualitative data, coding and recoding, and identification of categories and underlying themes. A framework of experiences across all domains was gen- erated, illustrated by participant quotations from all types of childless women in all domains of life. Deidentified quotations were attributed using identification numbers (denoted as ID), childlessness type and age. No more than one quotation from any participant was used.

\section{Results}

\subsection{Demographic Characteristics}

Table 1 shows participants' demographic characteristics and comparable Australian data. Compared with Australian females aged 25 to 44 years (89.4\%) (ABS, 2012b), 39.5 per cent fewer participants were married, in a civil union or de facto relationship (49.9\%; $n=386$; $95 \% \mathrm{Cl}$ for difference $-43.0--35.9)$. A higher percentage $(10.8 \%)$ of participants identified as lesbian or bisexual (13.0\%; $n=97)$ than Australian women aged 16 to 59 years $(2.2 \%$; $95 \% \mathrm{Cl}$ for difference $8.5-13.4$ ) (Smith, Rissel, Richters, Grulich, \& Visser, 2003). Compared with Australian women aged 15 years or over (19.9\%) (ABS, 2012b), 58.1 per cent more participants possessed a bachelor degree or higher $(78.0 \%$; $n=605$; $95 \% \mathrm{Cl}$ for difference $55.0-60.9)$. Almost half the participants $(48.5 \% ; n=361)$ were in the high-income bracket, compared with 20 per cent of Australian adults (95\% Cl for difference 24.9 - 32.1) (ABS, 2013). Close to three-quarters of participants $(73.2 \% ; n=554)$ identified as having no religion or being unsure, compared with 22.4 per cent of Australian females (ABS, 2012b). In contrast, only 21.0 per cent of participants identified as Christian, compared with 69.2 per cent of Australian females (ABS, 2012b).

Table 2 shows types of childless women and differences in demographic characteristics. There were differences between types of childless women's age ( $F=$ 51.8; $d f=4 ; p<0.001)$, relationship status $(\chi=97.6 ; d f$ $=4 ; p<0.001)$, sexual orientation $(\chi=13.1 ; d f=4 ; p=$ $0.01)$, religious status $(\chi=16.1 ; d f=4 ; p=0.003)$, area of geographic residence $(\chi=11.8 ; d f=4 ; p=0.02)$ and educational attainment $(H=20.1 ; d f=4 ; p<0.001)$.

\subsection{Indicators of Exclusion}

Table 3 shows indicators of exclusion in the social, civic, service and economic domains. Circumstantially childless women had lower social support scores than the undecided (Dunn's Test $=-97.0 ; p=0.001$ ), involuntarily childless (Dunn's Test $=114.1 ; p=0.004$ ), voluntarily childless (Dunn's Test $=-115.6 ; p<0.001$ ) and future childed (Dunn's Test $=-171.3 ; p<0.001$ ). While there was an overall difference in social interaction scores $(\mathrm{H}$ $=11.3 ; \mathrm{df}=4 ; \mathrm{p}=0.02$ ), post hoc testing identified no differences between types of childless women. 
Table 1. Demographic characteristics of participants and comparable Australian data.

\begin{tabular}{|c|c|c|c|}
\hline & $\begin{array}{l}\text { Participants } \\
\text { per cent }(n)\end{array}$ & Australian per cent & $\begin{array}{l}\text { Percentage difference } \\
(95 \% \mathrm{Cl})\end{array}$ \\
\hline \multicolumn{4}{|l|}{ Age $(n=775)$} \\
\hline $25-29$ & $25.4(197)$ & 24.8 & $0.6(-2.4-3.8)$ \\
\hline $30-34$ & $30.3(235)$ & 24.0 & $6.3(3.2-9.6)$ \\
\hline $35-39$ & $22.7(176)$ & 25.3 & $-2.6(-5.44-0.5)$ \\
\hline $40-44$ & $21.5(167)$ & $25.8^{1}$ & $-4.3(-7.0--1.3)$ \\
\hline \multicolumn{4}{|l|}{ Relationship status ( $\mathrm{n}=773$ ) } \\
\hline Single & $33.4(258)$ & Not available & \\
\hline Relationship (not living together) & $9.3(71)$ & Not available & \\
\hline De facto relationship & $26.4(204)$ & 35.7 & $-9.3(-12.3--6.1)$ \\
\hline Engaged & $5.4(42)$ & Not available & \\
\hline Married/civil union & $23.5(182)$ & $53.7^{2}$ & $-30.2(-33.1--27.1)$ \\
\hline Separated/divorced & $1.3(10)$ & 10.1 & $-8.8(-9.4--7.8)$ \\
\hline Widowed & $0.8(6)$ & $0.5^{1}$ & $0.3(-0.2-1.1)$ \\
\hline \multicolumn{4}{|l|}{ Sexual orientation $(n=745)$} \\
\hline Lesbian or bisexual & $13.0(97)$ & $2.2^{3}$ & $10.8(8.5-13.4)$ \\
\hline Heterosexual & $87.0(648)$ & 97.8 & $-10.8(-13.4--8.5)$ \\
\hline \multicolumn{4}{|l|}{ Educational attainment $(n=776)$} \\
\hline Year 12 or below & $5.0(39)$ & 58.6 & $-53.6(-55.0--51.9)$ \\
\hline Certificate/diploma & $17.0(132)$ & 21.5 & $-4.5(-7.0--1.8)$ \\
\hline Bachelor degree & $41.0(318)$ & 14.8 & $26.2(22.8-29.7)$ \\
\hline Graduate/postgraduate qualification & $37.0(287)$ & $5.1^{4}$ & $31.9(28.5-35.3)$ \\
\hline \multicolumn{4}{|l|}{ Employment status $(n=773)$} \\
\hline Employed/self-employed & $93.0(719)$ & 71.6 & $21.4(19.5-23.1)$ \\
\hline Unemployed & $6.3(49)$ & 3.9 & $2.4(0.9-4.3)$ \\
\hline Not in labour force & $0.6(5)$ & $24.5^{4}$ & $-23.9(-24.3--23.1)$ \\
\hline \multicolumn{4}{|l|}{ Personal income level $(n=744)$} \\
\hline Low (first three deciles) & $9.3(69)$ & 30.0 & $-20.7(-22.7--18.5)$ \\
\hline Lower middle (fourth decile) & $7.3(54)$ & 10.0 & $-2.7(-4.5--0.7)$ \\
\hline Middle (third quintile) & $7.4(55)$ & 20.0 & $-12.6(-14.3--10.6)$ \\
\hline Upper middle (fourth quintile) & $27.6(205)$ & 20.0 & $7.6(4.4-10.9)$ \\
\hline High (fifth quintile) & $48.5(361)$ & $20.0^{5}$ & $28.5(24.9-32.1)$ \\
\hline \multicolumn{4}{|c|}{ Aboriginal or Torres Strait Islander status $(n=771)$} \\
\hline Aboriginal or Torres Strait Islander & $1.4(11)$ & 2.5 & $-1.1(-1.7--0.03)$ \\
\hline Non-Aboriginal or Torres Strait Islander & $98.6(760)$ & $97.5^{1}$ & $1.1(0.03-1.8)$ \\
\hline \multicolumn{4}{|l|}{ Country of birth $(n=771)$} \\
\hline Born overseas & $14.1(109)$ & 26.2 & $-12.1(-14.4--9.5)$ \\
\hline Born in Australia & $85.9(662)$ & $73.8^{6}$ & $12.1(9.5-14.4)$ \\
\hline \multicolumn{4}{|l|}{ Language spoken at home $(n=772)$} \\
\hline Language other than English & $6.2(48)$ & 19.5 & $-13.3(-14.8--11.4)$ \\
\hline English only & $93.8(724)$ & $80.5^{6}$ & $13.3(11.4-14.8)$ \\
\hline \multicolumn{4}{|l|}{ Religion $(n=757)$} \\
\hline Christian & $21.0(159)$ & 69.2 & $-48.2(-51.0--45.2)$ \\
\hline Judaism & $0.4(3)$ & 0.5 & $-0.1(-0.4-0.6)$ \\
\hline Islam & $0.1(1)$ & 2.3 & $-2.2(-2.29--1.7)$ \\
\hline Indian religions & $1.8(14)$ & 4.2 & $-2.4(-3.1--1.2)$ \\
\hline Folk, new and other religions & $3.4(26)$ & 1.5 & $1.9(0.8-3.4)$ \\
\hline No religion or unsure & $73.2(554)$ & $22.4^{6}$ & $50.8(47.5-53.9)$ \\
\hline \multicolumn{4}{|l|}{ Geographic residence $(n=776)$} \\
\hline Major city & $77.4(601)$ & 68.4 & $9.0(6.0-11.9)$ \\
\hline Inner regional & $15.2(118)$ & 19.7 & $-4.5(-6.9--1.8)$ \\
\hline Outer regional & $6.4(50)$ & 9.5 & $-3.1(-4.6--1.2)$ \\
\hline Remote & $0.3(2)$ & 1.5 & $-1.2(-1.5--0.7)$ \\
\hline Very remote & $0.6(5)$ & $0.8^{7}$ & $-0.2(-0.6-0.6)$ \\
\hline
\end{tabular}

Notes: 1 Australian females aged 25 to 44 years (ABS, 2012b); 2 Registered marriages only (ABS, 2012b); 3 Australian females aged 16 to 59 years (Smith et al., 2003); 4 Australian females aged 15 years and over (ABS, 2012b); 5 Income deciles of Australian adults (ABS, 2013); 6 Australian females (ABS, 2012b); 7 Australian residents (ABS, 2008). 
Table 2. Demographic characteristics of types of childless women.

\begin{tabular}{|c|c|c|c|c|c|c|c|c|}
\hline & Involuntary & Circumstantial & Voluntary & Undecided & Future childed & $F^{1} / \chi^{2} / H^{3}$ & df & p value \\
\hline $\begin{array}{l}\text { Type of childless } \\
\text { women }(n=768)\end{array}$ & $\mathrm{n}=161$ & $n=161$ & $n=281$ & $n=139$ & $n=123$ & & & \\
\hline Per cent & $8.3 \%$ & $21.0 \%$ & $36.6 \%$ & $18.1 \%$ & $16.0 \%$ & & & \\
\hline Age in years $(n=767)$ & $\mathrm{n}=64$ & $n=161$ & $n=280$ & $n=139$ & $n=123$ & & & \\
\hline Mean & $\begin{array}{l}36.9 \\
(S D=4.9)\end{array}$ & $\begin{array}{l}36.5 \\
(S D=5.3)\end{array}$ & $\begin{array}{l}36.0 \\
(S D=5.6)\end{array}$ & $\begin{array}{l}31.7 \\
(S D=4.1)\end{array}$ & $30.1(S D=3.4)$ & $51.8^{1}$ & 4 & $<0.001$ \\
\hline $\begin{array}{l}\text { Relationship status } \\
(n=765)\end{array}$ & $n=64$ & $n=160$ & $n=281$ & $n=138$ & $n=122$ & & & \\
\hline Single & $17.2 \%$ & $64.4 \%$ & $31.0 \%$ & $13.1 \%$ & $13.1 \%$ & $97.6^{2}$ & 4 & $<0.001$ \\
\hline Partnered & $82.2 \%$ & $35.6 \%$ & $69.0 \%$ & $86.9 \%$ & $86.9 \%$ & & & \\
\hline $\begin{array}{l}\text { Sexual orientation } \\
(n=738)\end{array}$ & $n=62$ & $n=156$ & $n=272$ & $n=132$ & $n=116$ & & & \\
\hline Heterosexual & $90.3 \%$ & $91.0 \%$ & $81.3 \%$ & $88.6 \%$ & $91.4 \%$ & $13.1^{2}$ & 4 & 0.01 \\
\hline Lesbian or bisexual & $9.7 \%$ & $9.0 \%$ & $18.8 \%$ & $11.4 \%$ & $8.6 \%$ & & & \\
\hline Religion ( $n=765$ ) & $n=63$ & $\mathrm{n}=152$ & $n=277$ & $n=136$ & $n=122$ & & & \\
\hline Religious & $41.3 \%$ & $32.9 \%$ & $20.9 \%$ & $22.1 \%$ & $27.9 \%$ & $16.2^{2}$ & 4 & 0.003 \\
\hline Not religious/unsure & $58.7 \%$ & $67.1 \%$ & $79.1 \%$ & $77.9 \%$ & $72.1 \%$ & & & \\
\hline $\begin{array}{l}\text { Geographic residence } \\
(n=786)\end{array}$ & $n=64$ & $n=161$ & $n=281$ & $n=139$ & $n=123$ & & & \\
\hline Major city & $70.3 \%$ & $84.5 \%$ & $72.2 \%$ & $79.9 \%$ & $80.5 \%$ & $11.8^{2}$ & 4 & 0.02 \\
\hline Regional/ remote & $29.7 \%$ & $15.5 \%$ & $27.8 \%$ & $20.1 \%$ & $19.5 \%$ & & & \\
\hline $\begin{array}{l}\text { Educational } \\
\text { attainment }(n=768)\end{array}$ & $n=64$ & $n=161$ & $n=281$ & $n=139$ & $n=123$ & & & \\
\hline Year 11 or below & $0.0 \%$ & $1.2 \%$ & $2.1 \%$ & $1.4 \%$ & $0.0 \%$ & & & \\
\hline Year 12 or equivalent & $1.6 \%$ & $2.5 \%$ & $4.6 \%$ & $3.6 \%$ & $4.1 \%$ & & & \\
\hline $\begin{array}{l}\text { Certificate or } \\
\text { diploma }\end{array}$ & $20.3 \%$ & $14.9 \%$ & $23.8 \%$ & $12.2 \%$ & $8.9 \%$ & & & \\
\hline Bachelor or above & $78.1 \%$ & $81.4 \%$ & $69.4 \%$ & $82.7 \%$ & $87.0 \%$ & & & \\
\hline Mean rank & 388.4 & 398.3 & 352.0 & 402.2 & 418.8 & $20.1^{3}$ & 4 & $<0.001$ \\
\hline
\end{tabular}

Notes: 1 One Way ANOVA; 2 Chi Square Test for Independence; 3 Kruskal Wallis Analysis of Ranks.

Table 3. Indicators of exclusion in the social, civic, service and economic domains.

\begin{tabular}{|c|c|c|c|c|c|c|c|c|c|}
\hline & $\begin{array}{l}\text { All } \\
\text { participants }\end{array}$ & Involuntary & Circumstantial & Voluntary & Undecided & $\begin{array}{l}\text { Future } \\
\text { childed }\end{array}$ & $\mathrm{H}^{1}$ & df & $\begin{array}{l}P \\
\text { value }\end{array}$ \\
\hline Social interaction score ${ }^{2}$ & $n=765$ & $n=63$ & $\mathrm{n}=159$ & $n=276$ & $n=138$ & $n=121$ & & & \\
\hline Median & 7.0 & 7.0 & 7.0 & 7.0 & 8.0 & 8.0 & & & \\
\hline Mean rank & & 335.7 & 372.5 & 361.0 & 404.5 & 422.2 & 11.3 & 4 & 0.02 \\
\hline Social support score ${ }^{3}$ & $n=739$ & $n=60$ & $n=150$ & $n=273$ & $n=130$ & $n=119$ & & & \\
\hline Mean & $\begin{array}{l}55.4 \\
(S D=17.5)\end{array}$ & $\begin{array}{l}54.2 \\
(S D=22.2)\end{array}$ & $\begin{array}{l}46.1 \\
(S D=18.2)\end{array}$ & $\begin{array}{l}56.6 \\
(S D=17.3)\end{array}$ & $\begin{array}{l}56.3 \\
(S D=16.1)\end{array}$ & $\begin{array}{l}63.0 \\
(S D=12.3)\end{array}$ & & & \\
\hline Mean rank & & 383.1 & 269.0 & 384.5 & 366.0 & 440.3 & 49.0 & 4 & $<0.001$ \\
\hline $\begin{array}{l}\text { Community participation } \\
\text { score }^{4}\end{array}$ & $n=771$ & $n=64$ & $n=160$ & $n=278$ & $n=138$ & $n=123$ & & & \\
\hline Mean & $\begin{array}{l}3.2 \\
(S D=2.3)\end{array}$ & $\begin{array}{l}3.3 \\
(S D=2.8)\end{array}$ & $\begin{array}{l}3.3 \\
(S D=2.4)\end{array}$ & $\begin{array}{l}3.2 \\
(S D=2.3)\end{array}$ & $\begin{array}{l}3.3 \\
(S D=2.5)\end{array}$ & $\begin{array}{l}3.1 \\
(S D=2.0)\end{array}$ & & & \\
\hline Mean rank & & 373.7 & 386.1 & 386.0 & 379.0 & 375.3 & 0.4 & 4 & 0.98 \\
\hline $\begin{array}{l}\text { Problems accessing/using } \\
\text { services score } 5\end{array}$ & $n=776$ & $n=64$ & $\mathrm{n}=161$ & $n=261$ & $n=139$ & $n=123$ & & & \\
\hline Mean & $\begin{array}{l}70.7 \\
(S D=6.1)\end{array}$ & $\begin{array}{l}70.4 \\
(S D=6.6)\end{array}$ & $\begin{array}{l}70.3 \\
(S D=6.5)\end{array}$ & $\begin{array}{l}70.8 \\
(S D=6.3)\end{array}$ & $\begin{array}{l}69.9 \\
(S D=5.6)\end{array}$ & $\begin{array}{l}71.4 \\
(S D=5.1)\end{array}$ & & & \\
\hline Mean rank & & 363.2 & 372.6 & 394.4 & 382.2 & 391.2 & 1.8 & 4 & 0.77 \\
\hline Employment status & $n=773$ & $n=64$ & $n=159$ & $n=280$ & $n=139$ & $n=123$ & & & \\
\hline Employed & $83.3 \%$ & $87.5 \%$ & $82.4 \%$ & $81.8 \%$ & $82.0 \%$ & $87.8 \%$ & & & \\
\hline Underemployed & $9.7 \%$ & $6.3 \%$ & $9.4 \%$ & $10.4 \%$ & $10.1 \%$ & $8.9 \%$ & & & \\
\hline Unemployed & $6.3 \%$ & $4.7 \%$ & $8.2 \%$ & $6.8 \%$ & $7.2 \%$ & $3.3 \%$ & & & \\
\hline Not in labour force & $0.6 \%$ & $1.6 \%$ & $0.0 \%$ & $1.1 \%$ & $0.7 \%$ & $0.0 \%$ & & & \\
\hline Mean rank & & 398.4 & 378.5 & 376.9 & 377.5 & 401.0 & 3.6 & 4 & 0.5 \\
\hline Personal income levels & $n=744$ & $n=60$ & $\mathrm{n}=158$ & $n=267$ & $n=134$ & $\mathrm{n}=117$ & & & \\
\hline Low & $9.3 \%$ & $5.0 \%$ & $9.5 \%$ & $9.4 \%$ & $11.9 \%$ & $8.5 \%$ & & & \\
\hline Lower middle & $7.3 \%$ & $5.0 \%$ & $7.6 \%$ & $8.2 \%$ & $7.5 \%$ & $4.3 \%$ & & & \\
\hline
\end{tabular}




\begin{tabular}{|c|c|c|c|c|c|c|c|c|c|}
\hline & $\begin{array}{l}\text { All } \\
\text { participants }\end{array}$ & Involuntary & Circumstantial & Voluntary & Undecided & $\begin{array}{l}\text { Future } \\
\text { childed }\end{array}$ & $\mathrm{H}^{1}$ & df & $\begin{array}{l}P \\
\text { value }\end{array}$ \\
\hline Middle & $7.4 \%$ & $10.0 \%$ & $6.3 \%$ & $7.5 \%$ & $8.2 \%$ & $6.0 \%$ & & & \\
\hline Upper middle & $27.6 \%$ & $26.7 \%$ & $22.2 \%$ & $28.5 \%$ & $25.4 \%$ & $35.9 \%$ & & & \\
\hline High & $48.5 \%$ & $53.3 \%$ & $54.4 \%$ & $46.4 \%$ & $47.0 \%$ & $45.3 \%$ & & & \\
\hline Mean rank & & 392.6 & 384.8 & 360.0 & $355.9 \%$ & $367.9 \%$ & 3.0 & 4 & 0.6 \\
\hline
\end{tabular}

Notes: 1 Kruskal Wallis Analysis of Ranks; 2 Ordinal scale from 0 to 13. Higher scores indicate more social interaction; 3 Interval scale from 0 to 76 . Higher scores indicate greater levels of instrumental and affective social support; 4 Interval scale from 0 to 14 . Higher scores indicating participation in more community groups/events and activities; 5 Aggregate score from 5 to 75 . Lower scores indicating a greater degree of problems accessing or using service.

Table 4. Perceived stereotyping, stigmatisation and exclusion due to being childless.

\begin{tabular}{|c|c|c|c|c|c|c|c|c|c|}
\hline & $\begin{array}{l}\text { All partici- } \\
\text { pants } \\
(n=764)^{3}\end{array}$ & $\begin{array}{l}\text { Involuntary } \\
(n=64)^{4}\end{array}$ & $\begin{array}{l}\text { Circumstantial } \\
(n=160)^{5}\end{array}$ & $\begin{array}{l}\text { Voluntary } \\
(n=279)^{6}\end{array}$ & $\begin{array}{l}\text { Undecided } \\
(n=138)^{7}\end{array}$ & $\begin{array}{l}\text { Future } \\
\text { childed } \\
(n=123)^{8}\end{array}$ & $\mathrm{~F}^{1} / \mathrm{H}^{2}$ & df & $\begin{array}{l}P \\
\text { value }\end{array}$ \\
\hline \multicolumn{10}{|c|}{$\begin{array}{l}\text { Stigma consciousness } \\
\text { score }^{9}\end{array}$} \\
\hline Mean & $\begin{array}{l}36.6 \\
(S D=9.4)\end{array}$ & $\begin{array}{l}33.3 \\
(S D=8.6)\end{array}$ & $\begin{array}{l}34.1 \\
(S D=8.1)\end{array}$ & $\begin{array}{l}36.2 \\
(S D=9.0)\end{array}$ & $\begin{array}{l}37.9 \\
(S D=9.9)\end{array}$ & $\begin{array}{l}41.2 \\
(S D=9.5)\end{array}$ & $13.3^{1}$ & 4 & $<0.001$ \\
\hline \multicolumn{10}{|c|}{$\begin{array}{l}\text { Exclusion from social } \\
\text { interaction }{ }^{10}\end{array}$} \\
\hline Median & 5.0 & 4.0 & 4.0 & 5.0 & 5.0 & 5.0 & & & \\
\hline Mean rank & & 330.2 & 303.1 & 383.0 & 422.7 & 460.7 & $53.5^{2}$ & 4 & $<0.001$ \\
\hline \multicolumn{10}{|c|}{$\begin{array}{l}\text { Exclusion from social } \\
\text { support }^{10}\end{array}$} \\
\hline Median & 5.0 & 4.0 & 4.0 & 5.0 & 5.0 & 5.0 & & & \\
\hline Mean rank & & 355.5 & 305.1 & 382.7 & 424.0 & 450.2 & $46.7^{2}$ & 4 & $<0.001$ \\
\hline \multicolumn{10}{|c|}{$\begin{array}{l}\text { Exclusion from } \\
\text { community life }{ }^{10}\end{array}$} \\
\hline Median & 5.0 & 4.0 & 4.0 & 5.0 & 5.0 & 5.0 & & & \\
\hline Mean rank & & 303.9 & 310.6 & 407.9 & 402.7 & 433.0 & $49.3^{2}$ & 4 & $<0.001$ \\
\hline \multicolumn{10}{|c|}{ Exclusion from services ${ }^{10}$} \\
\hline Median & 5.0 & 5.0 & 5.0 & 5.0 & 5.0 & 5.0 & & & \\
\hline Mean rank & & 349.2 & 358.5 & 382.1 & 414.3 & 402.4 & $14.5^{2}$ & 4 & $<0.001$ \\
\hline \multicolumn{10}{|c|}{$\begin{array}{l}\text { Exclusion from material } \\
\text { and financial resources }{ }^{10}\end{array}$} \\
\hline Median & 5.0 & 5.0 & 5.0 & 5.0 & 5.0 & 5.0 & & & \\
\hline Mean rank & & 367.8 & 365.4 & 372.9 & 401.3 & 413.3 & $11.7^{2}$ & 4 & 0.02 \\
\hline \multicolumn{10}{|l|}{$\begin{array}{l}\text { Exclusion from } \\
\text { employment }{ }^{10}\end{array}$} \\
\hline Median & 5.0 & 5.0 & 5.0 & 5.0 & 5.0 & 5.0 & & & \\
\hline Mean rank & & 392.9 & 370.7 & 377.0 & 392.7 & 387.3 & $3.4^{2}$ & 4 & 0.5 \\
\hline
\end{tabular}

Notes: 1 One Way ANOVA; 2 Kruskal Wallis Analysis of Ranks; $3 \mathrm{n}=730$ for stigma consciousness and 763 for community life; $4 \mathrm{n}=150$ for stigma consciousness; $5 \mathrm{n}=150$ for stigma consciousness, 159 for community life and 161 for education; $6 \mathrm{n}=263$ for stigma consciousness, 278 for social interaction and employment, 281 for services and education, and 280 for material and financial resources; $7 \mathrm{n=132}$ for stigma consciousness, 137 for social interaction and 139 for education; $8 \mathrm{n}=117$ for stigma consciousness and 122 for employment and material and financial resources; 9 Aggregate index from 10 to 70, with lower scores indicating greater stigma consciousness; 10 Ordinal scale from 1 to 5 , with scores of 4 or below indicating at least a slight degree of exclusion due to being childless.

\subsection{Stigmatisation and Exclusion Due to Being Childless}

Table 4 shows stigmatisation and exclusion due to being childless. Future childed women's mean stigma consciousness score was higher than voluntarily, circumstantially and involuntarily childless women's scores $(p<0.001)$. Undecided women's mean stigma consciousness score was higher than circumstantially ( $p$ $=0.02$ ) and involuntarily ( $p=0.03$ ) childless women's scores. The results suggested future childed women per- ceived less stigmatisation due to being childless than voluntarily childless women, and future childed and undecided women perceived less stigmatisation than circumstantially and involuntarily childless women.

Participants perceived at least a slight degree of exclusion due to being childless from social interaction (47.3\%), social support (42.5\%), community life $(36.8 \%)$, services $(20.5 \%)$, material and financial resources $(17.7 \%)$ and employment $(12.2 \%)$. In relation to social interaction, circumstantially childless women 
perceived more exclusion than voluntarily childless (Dunn's test $=-79.9 ; p=0.001$ ), undecided (Dunn's test $=-119.6 ; p<0.001$ ) and future childed women (Dunn's test $=-157.6 ; p<0.001$ ), involuntarily childless women perceived more exclusion than undecided (Dunn's test $=-92.6 ; p=0.02$ ) and future childed women (Dunn's test $=-130.6 ; p<0.001$ ), and voluntarily childless women perceived more exclusion than future childed women (Dunn's test $=-77.7 ; p=0.004$ ). In relation to social support, circumstantially childless women perceived more exclusion than voluntarily childless (Dunn's test $=-77.6 ; p=0.001$ ), undecided (Dunn's test $=-118.9 ; p<0.001$ ) and future childed women (Dunn's test $=-145.1 ; p<0.001$ ), while involuntarily childless (Dunn's test $=-94.8 ; p=0.02$ ) and voluntarily childless women (Dunn's test $=-67.5 ; p=0.02$ ) perceived more exclusion than future childed women. Involuntarily and circumstantially childless women perceived more exclusion from community life than undecided (Dunn's test $=-98.7 ; p=0.006$ and Dunn's test $=-92.1 ; p<$ 0.001 ), voluntarily childless (Dunn's test $=-104.0 ; p=$ 0.001 and Dunn's test $=-97.3 ; p<0.001$ ) and future childed women (Dunn's test $=-129.1$ and $-122.4 ; p<$ 0.001). Circumstantially childless women perceived more exclusion from services than undecided women (Dunn's test $=-55.8 ; \mathrm{p}=0.02$ ).

\subsection{Experiences of Inclusion and Exclusion}

Childless women described pronatalism-driven experiences on a continuum of connection and inclusion within, exclusion within, and exclusion from, all domains of life. Each theme or element of a theme was experienced by all types of childless women in all domains of life, unless types of childless women and domains of life are specified. Some women described only connection and inclusion or exclusion, some reported connection and inclusion in some domains and exclusion in others, and some reported inclusion and exclusion in the same domain. Substantially more women reported exclusionary experiences within, than connection and inclusion within or exclusion from, the domains.

\subsubsection{Experiences of Inclusion and Connection}

Many women described experiences of inclusion and connection arising from their 'deviance' from pronatalism. Experiences of inclusion and connection are subtly different. 'Inclusion' refers to experiences in which childless women feel included by childed people and within childed domains of life. 'Connection' refers to experiences in which childless women have the resources and opportunities to connect and participate, despite the potential for exclusion by childed people or from childed domains of life.

Some women described experiences of inclusion.
For example, some involuntarily childless, circumstantially childless and future childed women received emotional and instrumental support, while some women valued being included in children's lives.

I am fortunate to have some friends around me who invite me to share in their families. I am a godmother and have regularly held nannying roles - it's a privilege to be entrusted with the responsibility of caring for people's kids and for them to tell me they know I'd treat them as if they were my own. (ID 224; undecided; age 32 years)

Furthermore, some voluntarily childless, undecided and future childed women reported others' understanding of their priorities and decisions.

My mother had a talk with me when I was in my early twenties. She said that she completely understood and agreed with my stance about being childfree and gave her full support. She told me that knowing me as well as she did, having children would be a mistake for me and I would probably always regret having them. (ID 125; voluntarily childless; age 40 years)

Other women described experiences of connection. For example, some women experienced affirmation of being childless through observations of or interactions with childed people.

Seeing overburdened mothers on public transport makes me grateful I don't have children. (ID 477; circumstantially childless; age 37 years)

Furthermore, many women valued their freedom to participate and contribute in all domains of life, and others actively built lives in which being childless was valued and affirmed.

I work full-time, but my husband works part-time in an unskilled job while he is studying for a more skilled job. I don't think we could have this arrangement if we had children. (ID 299; voluntarily childless; age 44 years)

I have a great group of friends and family who are like-minded.....Even if I had children I would not go to mother's groups ... because that wouldn't be my 'cuppa tea'. I like people who live outside of the box. (ID 117; circumstantially childless; age 42 years)

\subsubsection{Exclusionary Experiences within the Domains of} Life

Many women experienced being childless as a stigma- 
tising attribute, which resulted in exclusionary interpersonal interactions within domains of life, with friends, family, community members, service providers, clients and colleagues. For example, many women described others' assumptions and expectations they had children or would have children. When such expectations were not met, they experienced others' disappointment, invalidation of their worth and pressure to become childed. For example, some involuntarily childless women received unhelpful encouragement to become childed. Some circumstantially childless women felt pressured to settle for 'any man', and many circumstantially childless, undecided and future childed women were pressured to 'hurry up'. In addition, many voluntarily childless and undecided women described others' arguments they would feel different once they met the 'right man' or had children, were 'missing out', or were unqualified to decide not to have children. Voluntarily childless and undecided women also felt others pathologised their choices.

Many women also described being stereotyped and judged by others. Women who were perceived as making choices that contributed to being childless felt stereotyped as child-hating, career-focused, materialistic and selfish.

There is a perception you are selfish (so what if you are?!), a baby-hater, have something wrong with you or are ruthless. Your personality is judged by a decision you make about one aspect of your life. (ID 139; undecided; age 33 years)

All types of childless women felt judged as incomplete and unfulfilled. Involuntarily, circumstantially and voluntarily childless women who would not or could not ever become childed, felt further judged as defective and failed women. Some involuntarily and circumstantially childless women internalised such judgements.

The pressure to find a man and have a baby [as soon as possible] is huge. I feel it every single day of my life. There is no relief from feeling abnormal, shamed and like a failure. (ID 751; circumstantially childless; age 35 years)

Some involuntarily and circumstantially childless women who wanted children felt judged as unhappy and desperate. In contrast, some voluntarily childless, undecided and future childed women felt judged as abnormal and unnatural for not wanting, or not yet having, children. All except involuntarily childless women felt judged as culpable for not having or delaying children.

[W] hen I was 27 I was diagnosed with [polycystic ovarian syndrome] and was then informed by an older female [general practitioner] that I should have considered such diseases before I decided to be single and without a child. (ID 115; future childed; age 31 years)

Many women described others' beliefs they lacked knowledge, emotions, abilities and attributes that women acquired only upon becoming childed, including not understanding children and parenting, and being incapable of maturity, happiness, love, empathy and selflessness. Many women experienced invalidation of their views, contributions and expertise as a result of such beliefs.

We live in [an area] for private schools so there's always chatter about whose child goes where. I feel excluded from these discussions because I have no children but I also feel private schools suck money from public schools. Expressing that opinion is unpopular and is normally met with 'well you would think differently if you had kids'. No, I wouldn't. I find that assumption offensive. (ID 556; circumstantially childless; age 38 years)

Furthermore, many women felt others perceived their feelings, experiences and needs as less important than those of childed people, and accordingly prioritised the needs of childed people.

Not being granted leave during holiday periods to allow parents to take leave. Not having access to any flexible working arrangements (part time, work from home etc.), which are only granted to parents. Having to work back late to cover people leaving early to pick up kids from school. (ID 011; circumstantially childless; age 33 years)

In addition, many women experienced public questioning about their personal reasons for having no children, in response to which they felt required to justify, defend or conceal being childless. Finally, many women felt marginalised, invisible and irrelevant in interactions, activities and spaces dominated by childed people and children.

When I get together with my friends, they always talk about their children, and I sit there and have very minimal to say/input. I then tune out and feel lonely and very depressed. (ID 333; involuntarily childless; age 36 years)

\subsubsection{Exclusion from Childed Domains of Life}

Some women experienced exclusion from domains of life idealising, prioritising, dominated by or catering exclusively to childed people, or stigmatising childless women. In the social domain, some women experienced exclusion from financial and emotional support from family who prioritised childed relations, and many wom- 
en experienced exclusion from childed social networks.

Losing several very good friends to the world of 'mummyhood', even when you make all sorts of efforts to keep the relationship close it dies ... I feel these relationships would probably have survived and grown if I too had chosen to have children. (ID 119; voluntarily childless; age 36 years)

In the service domain, some circumstantially and voluntarily childless women were denied access to contraception and sterilisation procedures because medical practitioners believed they should become childed or would change their minds. Other women experienced exclusion from services that catered exclusively to childed people, or did not understand childless women's needs.

Gynaecological services for women not wishing to get pregnant are hard to access and [obstetric gynaecologists] seem uninterested in childfree woman and have a lack of knowledge about gynaecological problems outside of those relating to childbirth. (ID 017; voluntarily childless; age 35 years)

In the economic domain, some women experienced exclusion from employment and promotion opportunities by employers who assumed they would become childed, invalidated their expertise in working with children, or prioritised childed people's employment needs. Further, some involuntarily and circumstantially childless women described financial hardship arising from attempts to become childed, or following divorces from husbands who wanted to become childed.

Due to marital separation with husband after the stress of not having children my material and financial resources have suffered enormously. No longer have a home, struggle to make ends meet. (ID 218; involuntarily childless; age 32 years)

Finally, some women engaged in self-regulated exclusion from childed people, activities, groups, events and spaces, in order to avoid stigmatising experiences.

Went to appointment at [a hospital miscarriage clinic]. Same rooms and waiting rooms as pregnant women going for their check-ups. Indicated to sit at chairs facing wall and not towards the pregnant women ... I would have gone back to that clinic but it was too difficult due to so many other pregnant women around. (ID 087; involuntarily childless; age 33 years)

\section{Discussion}

This exploratory, mixed-methods study was the first to investigate the social exclusion of different types of childless women in their reproductive years, across multiple domains of life in Australian society. This discussion explores the nature and extent of social exclusion of childless women, differences in the social exclusion of types of childless women, and the study's strengths and limitations.

\subsection{The Nature and Extent of Social Exclusion of Childless Women}

The findings revealed a complex picture of the social exclusion of childless women. The qualitative and quantitative findings suggested childless women experienced pronatalism-driven stigmatisation, congruent with studies finding other people stereotyped and stigmatised childless women (Çopur \& Koropeckyj-Cox, 2010; Koropeckyj-Cox \& Pendell, 2007; LaMastro, 2001). The qualitative findings further suggested pronatalism manifested in a continuum of connection and inclusion within, exclusionary experiences within, and exclusion from, all domains of life. Some women felt being childless resulted in experiences of connection and the freedom to contribute and participate. However, many women experienced exclusion within the domains of life. For example, reflecting previous research that voluntarily childless women experienced pressure from family and friends to become childed (Rich et al., 2011), all types of childless women experienced such pressure in all domains of life. Many women were expected to justify being childless, supporting earlier findings that being childless was a discrediting attribute that women were required to justify or conceal in order to maintain credibility (Bell, 2013; Rich et al., 2011). In addition, confirming and expanding upon research finding childless women felt workplaces prioritised childed people's needs for annual and carers leave, flexible work and work-life balance, and that others doubted their professional credibility (Doyle et al., 2013; Rich et al., 2011), many women experienced subordination of their needs to those of childed people, assumptions they lacked qualities only childed women could possess, and discrediting of their views and expertise, in all domains of life. At the other end of the continuum, some childless women experienced exclusion from resources and participation in domains of life dominated by, prioritising or catering exclusively to childed people. This finding augments existing research revealing involuntarily childless women's exclusion from a childed social world (Loke, Yu, \& Hayter, 2012; Malik \& Coulson, 2013).

Supporting the qualitative findings, the quantitative findings revealed exclusion varied within and between the domains of life. In the social domain, childless women had moderate levels of social interaction and high levels of social support. While Italian research found that childless women were less likely to receive 
social support than childed women (Albertini \& Mencarini, 2014), it compared childless with childed women. In the civic domain, a new area of research for childless women in their reproductive years, childless women had low levels of participation in community groups, events and activities. In the service domain, childless women had a low degree of problems accessing or using services. However, in the context of qualitative findings that some women felt excluded from mental and reproductive health services, and a United States study finding 22 per cent of childless women had difficulty obtaining healthcare (Bernstein, 2001), the aggregate score may have disguised problems with particular services. In the economic domain, most women were employed and in the upper middle or high personal income brackets, in accordance with the weight of Australian and international research (Debest \& Mazuy, 2014; Miranti, McNamara, Tanton, \& Yap, 2009; Waren \& Pals, 2013).

In relation to perceived exclusion due to being childless, childless women perceived more exclusion in the social and civic domains than the service and economic domains. This may be explained by the implications of pronatalism in different domains. For example, stigmatising experiences may be more salient in the social and civic domains, while freedom to participate may be more pertinent to the service and economic domains. In addition, when considering the continuum of connection and inclusion within, exclusionary experiences within, and exclusion from, domains of life, the qualitative findings revealed substantially more women described exclusionary experiences within domains of life, than experiences of connection and inclusion within, or exclusion from, domains of life. This facilitates an understanding of why the quantitative findings did not consistently reveal exclusion from, or connection and inclusion within, domains of life, by suggesting exclusionary experiences within domains of life are more relevant to childless women.

\subsection{Differences in Social Exclusion of Types of Childless Women}

The findings revealed an equally complex picture of exclusion of types of childless women. In relation to the indicators of exclusion, circumstantially childless women reported lower levels of social support than other types of childless women. There were no statistically significant differences in other indicators of exclusion, suggesting different types of childless women had similar levels of resources and participation. Australia's divergent cultural, social, political and economic context from other countries may explain inconsistencies with United States and French studies, which found voluntarily childless women had higher workforce participation rates and incomes than other childless women (Abma \& Martinez, 2006; Waren \& Pals, 2013). In rela- tion to perceived exclusion due to being childless, circumstantially and involuntarily childless women, followed by voluntarily childless women, perceived more exclusion than undecided and future childed women. These differences corresponded with findings that involuntarily, circumstantially and voluntarily childless women perceived more stigmatisation due to being childless than future childed women, and involuntarily and circumstantially childless women perceived more stigmatisation than undecided women.

The qualitative findings provided additional evidence of nuanced differences in childless women's experiences of inclusion and exclusion. Such differences were influenced by whether women's 'deviance' from pronatalism was non-volitional (innocent), volitional (guilty), and temporary or permanent. For example, some involuntarily and circumstantially childless women, for whom being childless was non-volitional, received sympathy and support. However, others felt stereotyped as unhappy and desperate, consistent with previous research with involuntarily childless women (McCarthy, 2008). In contrast, women whom others perceived as culpable for choices that 'contributed' to being childless, felt stereotyped as selfish, materialistic and child-hating. Furthermore, women who were permanently childless felt judged as failures. In addition, some circumstantially childless and voluntarily childless women who were permanently childless, and undecided women who were considering becoming permanently childless, experienced divorce or separation from partners who wanted to become childed, building upon earlier research with involuntarily childless women (Bell, 2013).

The findings also supported earlier studies revealing childless women's agency (Doyle et al., 2013; Park, 2002), by suggesting childless women are not simply passive receivers of social exclusion. Rather, their internalised, disempowered or empowered responses influenced experiences of social exclusion. Expanding upon research with involuntarily childless women (Bell, 2013), some involuntarily and circumstantially childless women who perceived themselves as failures, incomplete and unfulfilled, were deeply cognisant of being excluded. Furthermore, while some women inadvertently exacerbated exclusion by avoiding stigmatising experiences, others made empowered choices to build lives in which being childless was valued and affirmed.

The influence of the nature of women's 'deviance' from pronatalism facilitates an understanding of differences in types of childless women's degree of perceived exclusion. Some involuntarily and circumstantially childless women's greater degree of exclusion may have been influenced by permanent, internalised and disempowered non-conformance, despite involuntarily childless women's 'innocent' deviance. In contrast, some voluntarily childless women may have been protected from their volitional ('culpable') and perma- 
nent deviance by empowered choices. Finally, some undecided and future childed women may have been protected by temporary non-conformance with pronatalism, whereby they were included on the basis of assumptions they would become childed in future.

\subsection{Strengths and Limitations}

The study had a number of limitations. In relation to the qualitative component, neither iterative data collection and analysis nor participant validation was possible in the context of an anonymous questionnaire. In relation to the quantitative component, its crosssectional design precluded an examination of causality. Selection bias may have been introduced by the fiftyfive per cent completion rate, and non-probability sampling limited external validity. Although selfselection was unavoidable due to the absence of a sampling frame, it may have produced a sample that was more likely to experience pronatalism-driven stigmatisation and exclusion. While the sample was not comparable with Australian women, participants' high educational attainment, employment rates, personal incomes and proportion of voluntary childlessness were consistent with research on the characteristics of childless women (Abma \& Martinez, 2006; Miranti et al., 2009; Waren \& Pals, 2013). Finally, due to its exploratory nature, the study did not control for confounding factors.

Despite its limitations, this study was the first to investigate social exclusion in multiple domains of life of different types of childless women in their reproductive years. Furthermore, the mixed methods approach facilitated a deeper understanding of social exclusion, the large sample provided sufficient power and rich qualitative data, the use of existing and validated scales reduced the potential for measurement bias, and self-administration minimised socially desirable responses. In addition, data immersion, participant quotations, locating the findings within existing research, and researcher reflexivity enhanced the qualitative component's rigour.

\section{Conclusions}

This research suggests societal-level pronatalism manifests in a continuum of childless women's connection and inclusion within, exclusion within, and exclusion from domains of life in Australian society, with more exclusion in the social and civic domains than the service and economic domains. Future research using a more comprehensive suite of indicators will further elucidate the nature of social exclusion. The study further suggests circumstantially and involuntarily childless women, followed by voluntarily childless women, perceive more stigmatisation and exclusion due to being childless than undecided and future childed wom- en. Such differences may be influenced by the nature of women's 'deviance' from pronatalism.

Given social exclusion is a firmly established social determinant of health, it is essential to work towards an Australian society in which women's motherhood status is irrelevant to judgements of their worth, such that none would feel excluded in connection with being childless. Inclusive research can contribute to this change by measuring and exploring motherhood status and types of childlessness in health and social research. In addition, practitioners and policy-makers can develop inclusive interventions and policies by ensuring childless women's needs are considered in interventions and policies for all women, directly targeting different types of childless women with support groups, activities and events, and constructing counterdiscourses of being childless as a positive and valued attribute.

\section{Acknowledgements}

The authors thank the women who participated in this research.

\section{Conflict of Interests}

The authors declare no conflict of interests.

\section{References}

Abma, J. C., \& Martinez, G. M. (2006). Childlessness among older women in the United States: Trends and profiles. Journal of Marriage and Family, 68(4), 1045-1056.

Australian Bureau of Statistics. (2007). 2006 Census community profile series: Basic community profile: Australia. Canberra: ABS.

Australian Bureau of Statistics. (2008). Australian social trends, 2008. Canberra: ABS.

Australian Bureau of Statistics. (2010a). Australian social trends, Dec 2010. Canberra: ABS.

Australian Bureau of Statistics. (2010b). General social survey. Canberra: ABS.

Australian Bureau of Statistics. (2011). Census household form. Canberra: ABS.

Australian Bureau of Statistics. (2012a). 2011 Census of population and housing: Basic community profile: Australia. Canberra: ABS.

Australian Bureau of Statistics. (2012b). Australia 2011 Census of population and housing: Basic community profile. Canberra: ABS.

Australian Bureau of Statistics. (2012c). Income data in the census. Retrieved from http://www.abs.gov.au/ websitedbs/censushome.nsf/home/factsheetsuid? opendocument $\&$ navpos $=450$

Australian Bureau of Statistics. (2013). Australian health survey: Users' guide, 2011-13. Canberra: ABS. 
Albertini, M., \& Mencarini, L. (2014). Childlessness and support networks in later life new pressures on familistic welfare states? Journal of Family Issues, 35(3), 331-357.

Australian Institute of Health and Welfare. (2010). Australia's mothers and babies 2008. Canberra: AlHW.

Baker, M. (2003). Infertility, social exclusion and social policy. Paper presented at the Australian Social Policy Conference, Sydney.

Bell, K. (2013). Constructions of "infertility" and some lived experiences of involuntary childlessness. Affilia, 28(3), 284-295.

Bernstein, A. B. (2001). Motherhood, health status, and health care. Women's Health Issues, 11(3), 173-184.

Brown, B., Brauner, C., Chan, A., Gutierrez, D., Herson, J., Lovato, J., ... Vienier, J. (2010). STPLAN Version 4.5: February, 2010: Calculations for sample sizes and related problems. Houston, Texas: University of Texas M.D. Anderson Cancer Center.

Butterfield, L, Borgen, W, Amundson, N., \& Maglio, A. (2005). Fifty years of the critical incident technique: 1954-2004 and beyond. Qualitative Research, 5(4), 475-497.

Cannold, L. (2005). What, no baby? Why women are losing the freedom to mother, and how they can get it back. Fremantle: Curtin University Books.

Çopur, Z., \& Koropeckyj-Cox, T. (2010). University students' perceptions of childless couples and parents in Ankara, Turkey. Journal of Family Issues, 31(11), 1481-1506.

Daniluk, J. C. (2001). Reconstructing their lives: a longitudinal, qualitative analysis of the transition to biological childlessness for infertile couples. Journal of Counseling \& Development, 79(4), 439-449.

Debest, C., \& Mazuy, M. (2014). Childlessness: A life choice that goes against the norm. Population \& Societies, 508, 1-4.

Dermott, E., Fahmy, E., Gordon, D., Heslop, P., Levitas, R., \& Patsios, D. (2012). Poverty and social exclusion in the UK: Living standards questionnaire 2012. Bristol: PSE.

Doyle, J., Pooley, J. A., \& Breen, L. (2013). A phenomenological exploration of the childfree choice in a sample of Australian women. Journal of Health Psychology, 18(3), 397-407.

Fieder, M., Huber, S., \& Bookstein, F. L. (2011). Socioeconomic status, marital status and childlessness in men and women: An analysis of census data from six countries. Journal of Biosocial Science, 43(05), 619635.

George, L., Blazer, D., Hughes, D., \& Fowler, N. (1989). Social support and the outcome of major depression. The British Journal of Psychiatry, 154(4), 478-485.

Gillespie, R. (2000). When no means no: Disbelief, disregard and deviance as discourses of voluntary childlessness. Women's Studies International Forum, 23(2), 223-234.
Graham, M., \& Rich, S. (2012a). Representations of childless women in the Australian print media. Feminist media studies, 14(3), 500-518.

Graham, M., \& Rich, S. (2012b) What's 'childless' got to do with it? Geelong: Alfred Deakin Research Institute.

Hammarberg, K., Astbury, J., \& Baker, H. (2001). Women's experience of IVF: A follow-up study. Human Reproduction, 16(2), 374-383.

Huber, S., Bookstein, F. L., \& Fieder, M. (2010). Socioeconomic status, education, and reproduction in modern women: An evolutionary perspective. American Journal of Human Biology, 22(5), 578-587.

Hughes, D., Blazer, D., \& Hybels, C. (1990). Duke social support index (DSSI): A working paper (revised). Durham, NC: Duke University Medical Center.

Koropeckyj-Cox, T., \& Pendell, G. (2007). Attitudes about childlessness in the United States: Correlates of positive, neutral, and negative responses. Journal of Family Issues, 28(8), 1054-1082.

LaMastro, V. (2001). Childless by choice? Attributions and attitudes concerning family size. Social Behavior and Personality, 29(3), 231-243.

Leech, N. L., \& Onwuegbuzie, A. J. (2009). A typology of mixed methods research designs. Quality \& Quantity, 43(2), 265-275.

Letherby, G. (1999). Other than mother and mothers as others: The experience of motherhood and nonmotherhood in relation to "infertility" and "involuntary childlessness". Women's Studies International Forum, 22(3), 359-372.

Levitas, R., Pantazis, C., Fahmy, E., Gordon, D., Lloyd, E., \& Patsios, D. (2007). The multi-dimensional analysis of social exclusion. London: Cabinet Office.

Loke, A. Y., Yu, P.-L., \& Hayter, M. (2012). Experiences of sub-fertility among Chinese couples in Hong Kong: A qualitative study. Journal of Clinical Nursing, 21(3/4), 504-512.

Malik, S. H., \& Coulson, N. S. (2013). Coming to terms with permanent involuntary childlessness: A phenomenological analysis of bulletin board postings. Europe's Journal of Psychology, 9(1), 77-92.

McCarthy, M. P. (2008). Women's lived experience of infertility after unsuccessful medical intervention. Journal of Midwifery \& Women's Health, 53(4), 319 324.

McNamee, B. G., \& James, G. D. (2012). The impact of child-rearing status on perceptual and behavioural predictors of ambulatory blood pressure variation among working women. Annals of Human Biology, 39(6), 490-498.

Melbourne Institute of Applied Economic and Social Research. (2012). Household, income and labour dynamics in Australia (HILDA) survey: Wave 12M new person questionnaire. Melbourne: Melbourne Institute of Applied Economic and Social Research.

Miall, C. E. (1994). Community constructs of involuntary 
childlessness: Sympathy, stigma, and social support. Canadian Review of Sociology, 31(4), 392-421.

Miranti, R., McNamara, J., Tanton, R., \& Yap, M. (2009). A narrowing gap? Trends in the childlessness of professional women in Australia 1986-2006. Journal of Population Research, 26(4), 359-379.

Mollen, D. (2006). Voluntarily childfree women: Experiences and counseling considerations. Journal of Mental Health Counseling, 28(3), 269-282.

Onat, G., \& Beji, N. K. (2012). Marital relationship and quality of life among couples with infertility. Sexuality and Disability, 30(1), 39-52.

Park, K. (2002). Stigma management among the voluntarily childless. Sociological Perspectives, 45(1), 2145.

Parry, D. (2004). Women's lived experiences of infertility. Canadian Woman Studies, 24(1), 81-86.

Pinel, E. C. (1999). Stigma consciousness: The psychological legacy of social stereotypes. Journal of Personality and Social Psychology, 76(1), 114-128.

Popay, J., Escorel, S., Hernández, M., Johnston, H., Mathieson, J., \& Rispel, L. (2008). Understanding and tackling social exclusion: Final report to the WHO Commission on Social Determinants of Health from the Social Exclusion Knowledge Network. Lancaster,
UK: WHO Social Exclusion Knowledge Network.

Rich, S., Taket, A., Graham, M., \& Shelley, J. (2011). 'Unnatural', 'unwomanly', 'uncreditable' and 'undervalued': The significance of being a childless woman in Australian society. Gender Issues, 28(4), 226-247.

Sawer, M. (2013). Misogyny and misrepresentation women in Australian parliaments. Political Science, 65(1), 105-117.

Sherbourne, C. D., \& Stewart, A. L. (1991). The MOS social support survey. Social Science and Medicine, 32(6), 705-714.

Smith, A., Rissel, C. E., Richters, J., Grulich, A. E., \& Visser, R. O. (2003). Sex in Australia: Sexual identity, sexual attraction and sexual experience among a representative sample of adults. Australian and New Zealand Journal of Public Health, 27(2), 138-145.

Sternke, E. A., \& Abrahamson, K. (2015). Perceptions of women with infertility on stigma and disability. Sexuality and Disability, 33(1), 3-17.

Veevers, J. E. (1979). Voluntary childlessness. Marriage \& Family Review, 2(2), 1-26.

Waren, W., \& Pals, H. (2013). Comparing characteristics of voluntarily childless men and women. Journal of Population Research, 30(2), 151-170.

\section{About the Authors}

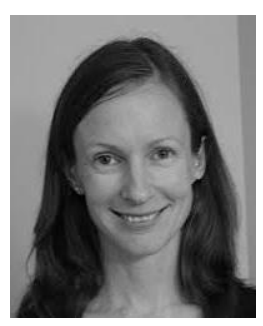

\section{Beth Turnbull}

Beth Turnbull is a PhD candidate in the School of Health and Social Development, Deakin University. Her research seeks to understand the social connection and exclusion in different areas of life of women with no children, and how those experiences influence health and wellbeing.

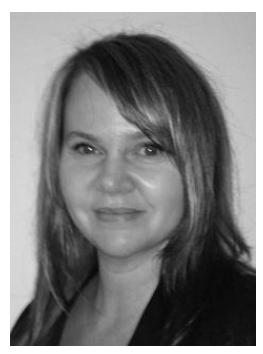

\section{Dr. Melissa Graham}

Melissa Graham is a Senior Lecturer in the School of Health and Social Development, Deakin University. She is the Deputy Director of the Centre for Health through Action on Social Exclusion (CHASE). Dr Graham's research explores the lives of women without children with a particular focus on social exclusion, and health and wellbeing.

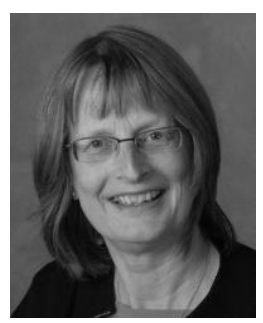

\section{Ann Taket}

Ann Taket holds the chair in Health and Social Exclusion within the School of Health and Social Development, and is Director of the Centre for Health through Action on Social Exclusion (CHASE) at Deakin University. Professor Taket has over thirty years' experience in public health related research, with particular interests in research directed at understanding the complex interactions between social exclusion and health, prevention and intervention in violence and abuse and the value of human rights based approaches in policy and practice. 
Appendix: Open-ended questions used in the data collection instrument.

\begin{tabular}{|c|c|}
\hline Topic/domain & Question \\
\hline \multirow[t]{2}{*}{ Stigmatisation } & Can you think of a time when you felt you felt you were stereotyped or stigmatized because \\
\hline & you have no children? If so, please describe the incident in the space provided. \\
\hline \multirow[t]{2}{*}{ Social domain } & $\begin{array}{l}\text { Thinking about your family and friends, can you think of a time when you had a positive or } \\
\text { negative experience related to not having children? If so, please describe the incident in detail } \\
\text { in the space provided. }\end{array}$ \\
\hline & $\begin{array}{l}\text { Thinking about your participation in social and leisure activities, can you think of a time when } \\
\text { you had a positive or negative experience related to not having children? If so, please describe } \\
\text { the incident in detail in the space provided. }\end{array}$ \\
\hline Civic domain & $\begin{array}{l}\text { Thinking about your participation in community life, can you think of a time when you had a } \\
\text { positive or negative experience related to not having children? If so, please describe the } \\
\text { incident in detail in the space provided. }\end{array}$ \\
\hline Service domain & $\begin{array}{l}\text { Thinking about your access to and use of services, can you think of a time when you had a } \\
\text { positive or negative experience related to not having children? If so, please describe the } \\
\text { incident in detail in the space provided. }\end{array}$ \\
\hline \multirow[t]{2}{*}{ Economic domain } & $\begin{array}{l}\text { Thinking about your working life (including paid work and unemployment), can you think of a } \\
\text { time when you had a positive or negative experience related to not having children? If so, } \\
\text { please describe the incident in detail in the space provided. }\end{array}$ \\
\hline & $\begin{array}{l}\text { Thinking about your material and financial resources, can you think of a time when you had a } \\
\text { positive or negative experience related to not having children? If so, please describe the } \\
\text { incident in detail in the space provided. }\end{array}$ \\
\hline General & $\begin{array}{l}\text { Is there anything else you would like to tell us about being a woman in Australian society who } \\
\text { does not have children? }\end{array}$ \\
\hline
\end{tabular}

